\title{
Effect of Unilateral Transcranial Direct Current Stimulation on Reaction Time in Veterans and Athletes with Disabilities
}

\section{ART ICLE INF O}

\section{Article Type}

Original Research

\section{Authors}

Arastoo A.A. ${ }^{1} P h D$

Parsaei S. ${ }^{* 2} M S c$,

Zahednejad Sh. ${ }^{3} \mathrm{PhD}$,

Alboghebish S. ${ }^{2} M S c$,

BurBur A. ${ }^{4} M S C$

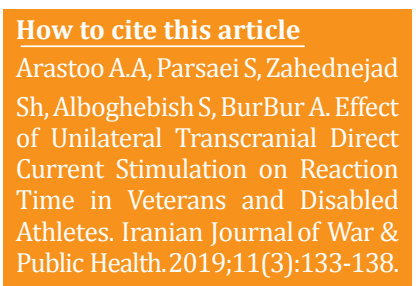

${ }^{1 " S o c i a l ~ F a c t o r s ~ A f f e c t i n g ~ H e a l t h ~ R e-~}$ search Center" and "Musculoskeletal Rehabilitation Research Center" and "Public Health Department, Health Faculty", Ahvaz Jundishapur University of Medical Sciences, Ahvaz, Iran ${ }^{2}$ Sports Psychology Department, Physical Education Faculty, Shahid Chamran University of Ahvaz, Ahvaz, Iran

3"Musculoskeletal Rehabilitatio Research Center" and "Physical Therapy Department, Rehabilitation Faculty", Ahvaz Jundishapur University of Medical Sciences, Ahvaz, Iran ${ }^{4}$ Physical Education Department, Literature \& Human Sciences Faculty, Farhangian University, Tehran, Iran

\section{*Correspondence}

Address: Sports Psychology Department, Physical Education \& Sport Sciences Faculty, Shahid Chamran University of Ahvaz, Golestan Boulevard, Ahvaz, Iran. Postal Code: 6135783511

Phone: +98 (84) 35724587

Fax: +98 (84) 35724587

sajadparsaei93@gmail.com

\section{Article History}

Received: September 29, 2018

Accepted: March 25, 2019

ePublished: July 21, 2019

\section{A B S T R A C T}

Aims Recently, Transcranial Direct Current Stimulation (tDCS) has been considered by researchers to improve various processes. The aim of this study was to investigate the effect of tDCS on reaction time in veterans and athletes with disabilities.

Materials \& Methods This semi-experimental study with pre-test post-test design was conducted in 2018 among all veterans and athletes with disabilities, who were members of Veterans and disabled board of Shiraz. 24 veterans and disabled persons of Shiraz athletes were selected by available sampling and allocated to experimental and artificial stimulation (sham) groups. Acquisition stage was held during 3 sessions. In the experimental group, an anode electrode was placed on the $\mathrm{C} 4$ and the cathode electrode was placed in the FP1. The stimulation rate was 1.5 milliamps in 20 minutes. In the sham groups, the anode and cathode electrode were placed on the C4 and FP1 points, but the stimulation was discontinued after 30 seconds. After the last session, a post-test was performed. Data analysis was performed by SPSS 22, using independent t-test and Multivariable analysis of covariance.

Findings In the post-test, there was a significant difference between the experimental group and the sham at the simple reaction time and choice reaction time and the performance of the experimental group was better than the sham group $(\mathrm{p}=0.0001)$.

Conclusion tDCS can improve the simple and choice reaction time in veterans and athletes with disabilities.

Keywords Electrical Stimulation of the Brain; Reaction Time; Veterans; Disabled

\section{CIT A T I O N L I N K S}

[1] Effect of a period of selected SMR/Theta neurofeedback training on visual ... [2] Effects of the amount and intensity of exercise on ... [3] Comparison of self-regulation components between the disabled ... [4] Developing the World Health ... [5] An exploration of the viability and usefulness of the construct ... [6] Comparing the mental health of the athletic and non ... [7] Effectiveness of physical activity on quality of life and pain selfefficacy ... [8] The serial reaction time task: Implicit motor ... [9] The effect of cognitive and motivational imagery on choice ... [10] Motor learning and performance: From principles ... [11] On the reduction of reaction time with mental ... [12] Transcranial Direct Current Stimulation (tDCS) produces localized and specific alterations in ... [13] A systematic review on reporting and assessment of adverse effects associated with transcranial direct ... [14] An introduction to neurotechnologies, transcranial magnetic stimulation and transcranial direct current ... [15] tDCS-induced effects on executive functioning and their ... [16] Investigating the role of current strength in tDCS modulation of ... [17] The effect of transcranial direct current stimulation on working memory ... [18] The effects of transcranial direct current stimulation on mental ... [19] Bilateral tDCS on primary motor cortex: Effects ... [20] Assiament facilitate and significant interference of Stroop ... [21] Anodal direct current stimulation in the healthy aged: Effects ... [22] Comparison between auditory and visual simple ... [23] Effect of transcranial direct current stimulation over the primary motor cortex ... [24] Introducing graph theory to track for neuroplastic alterations in the resting human brain: A transcranial ... [25] tDCS possibly stimulates glial ... [26] The role of the dorsal medial frontal cortex in central processing limitation: A transcranial ... [27] Quality of life in chemical weapon victims 15 years after exposure ... [28] Access to green space, physical activity and mental health ... 


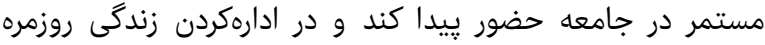

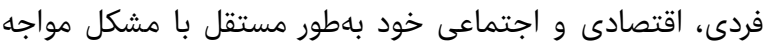

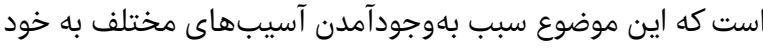

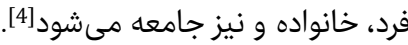

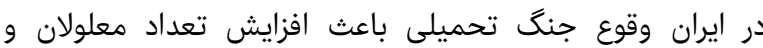

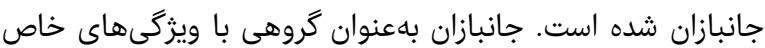

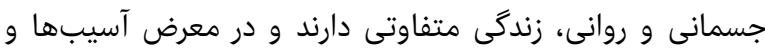

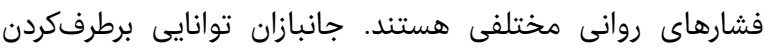

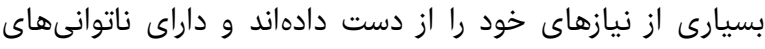

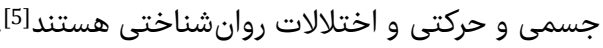

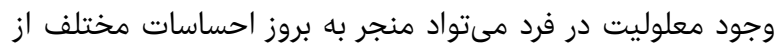

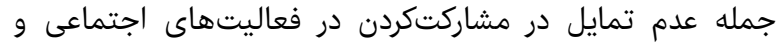

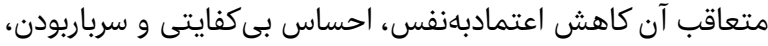

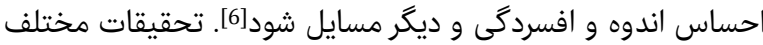

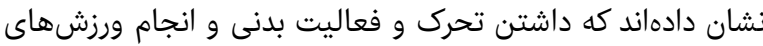

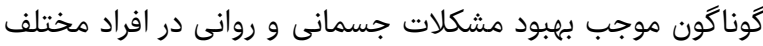

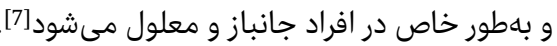

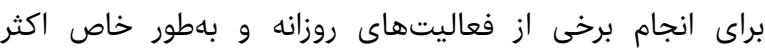

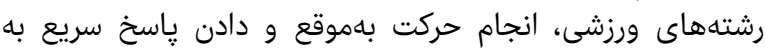

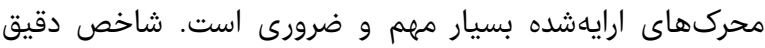

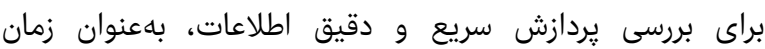

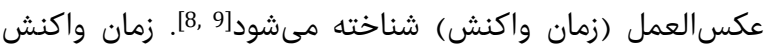

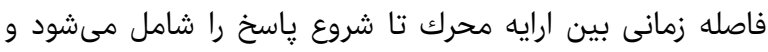

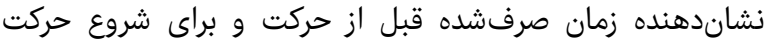

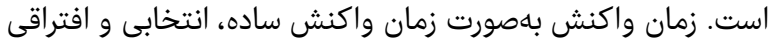

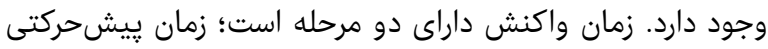

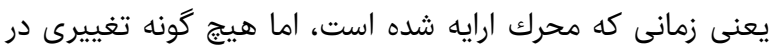

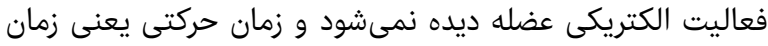

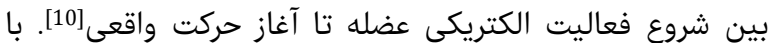

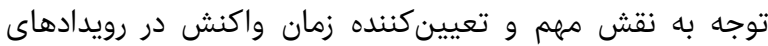

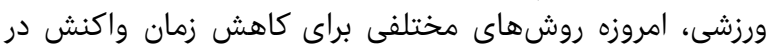

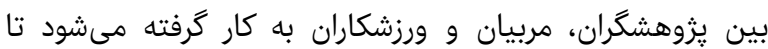

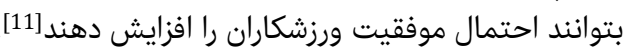

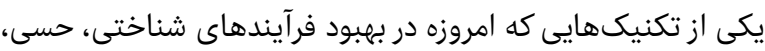

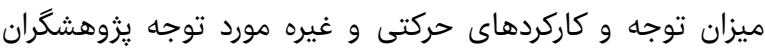

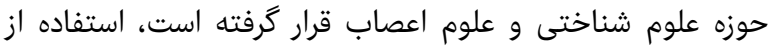

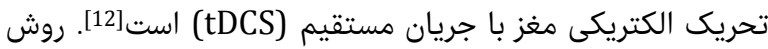

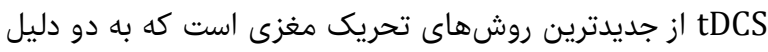

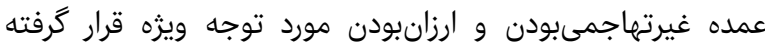

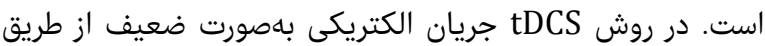

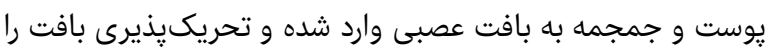

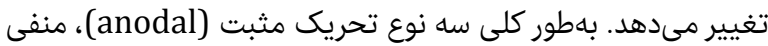

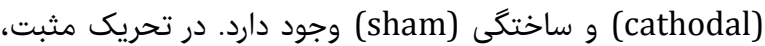

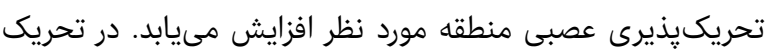

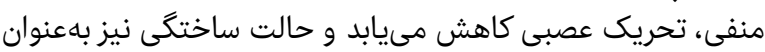

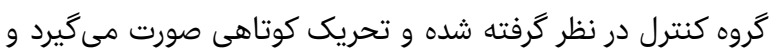

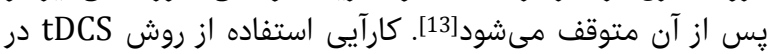

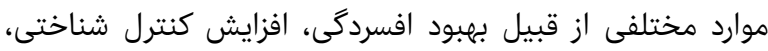

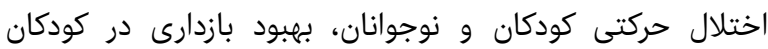

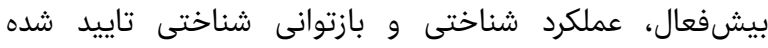

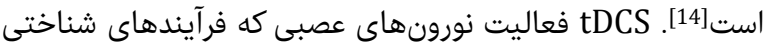

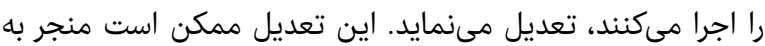

\section{تاثير تحريك الكتريكى فراجمجمهاى يكارطرفه مغز بر زمان واكنش جانبازان و ورزشكاران بانيان

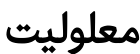

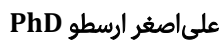

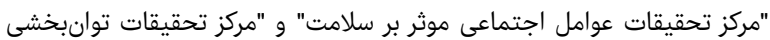

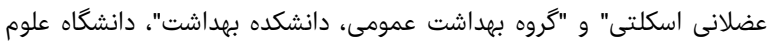

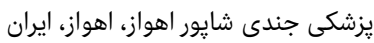

MSc سجاد بارسايى

كروه روانشناسى ورزش، دارسايى انشكده تربيت بدنى، دانشكاه شهيد جمران اهواز،

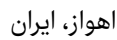

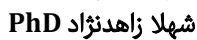

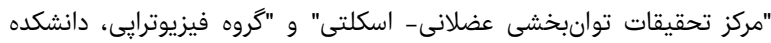

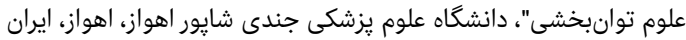

MSc سعيد البوغبيش توران

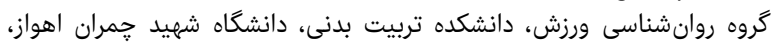

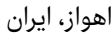

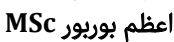
كروه تربيتبدنى، دانشكده ادبيات و علوم انسانى، دانشكاه فرهنگيان، تهران، ايران

جكيده

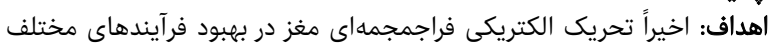

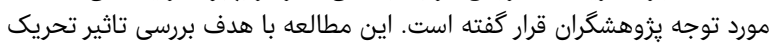

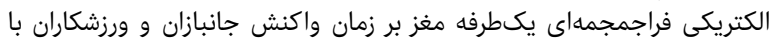

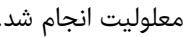

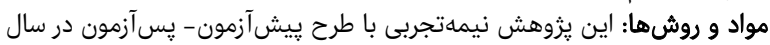

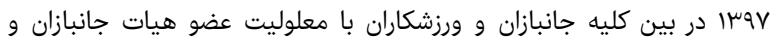

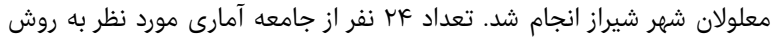

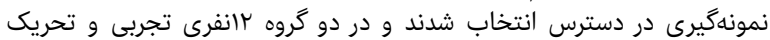

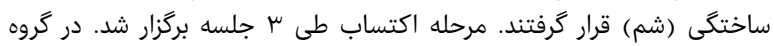

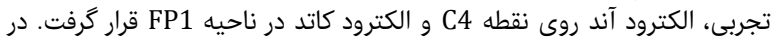

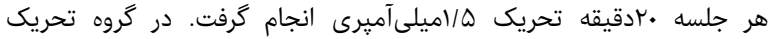

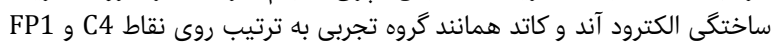

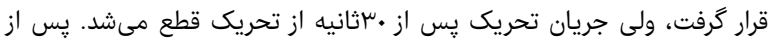

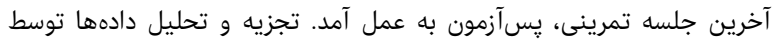

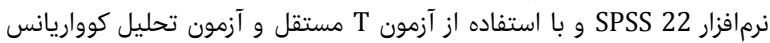
جندمتغيره انجام شد.

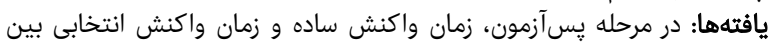

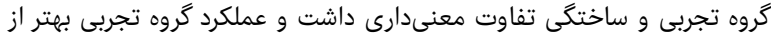

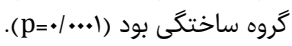

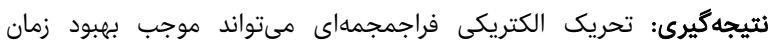

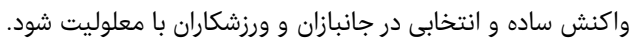

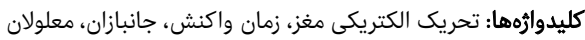

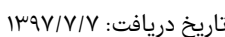

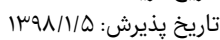

sajadparsaei93@gmail.com :نويسنده مسئول: تورل

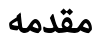

وجود افراد معلول و كمتوان در همه دورههاى زندگى بشر بـ بهصورت

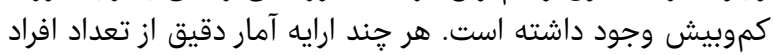

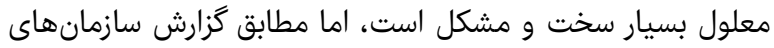

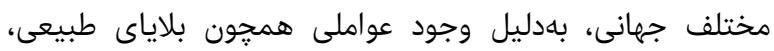

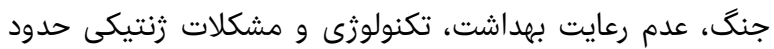

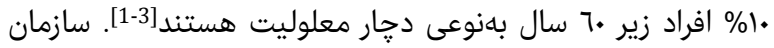

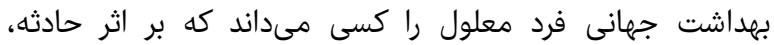

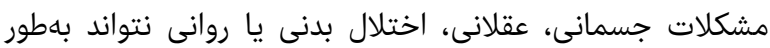




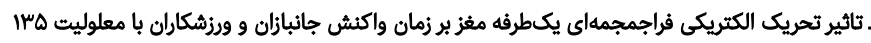

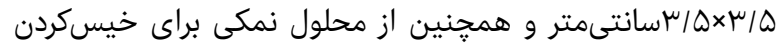

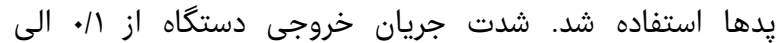

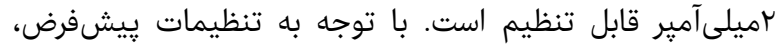

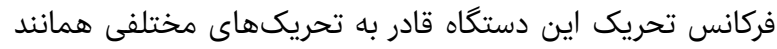

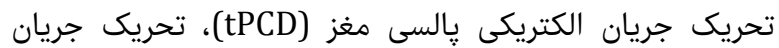

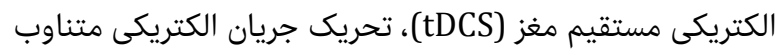

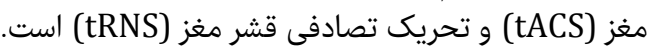

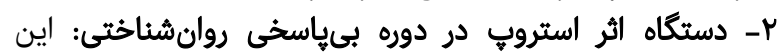

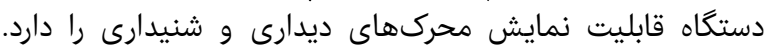

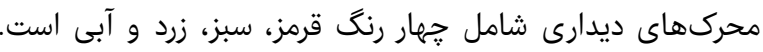

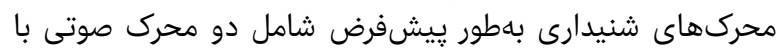

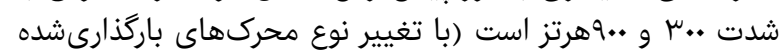

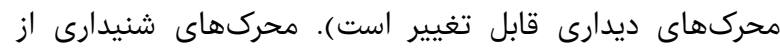
طريق هدفون و محركهاى ديدارى از طريق صفايل تغدير است نمايشكر رايانه

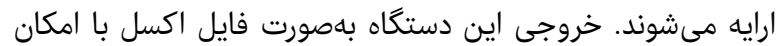

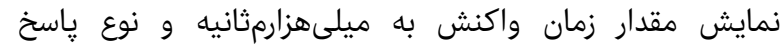

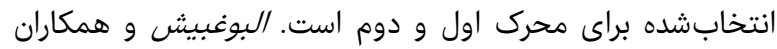

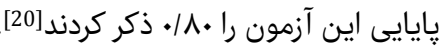

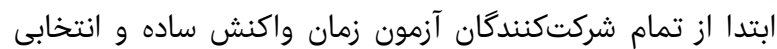

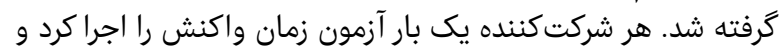

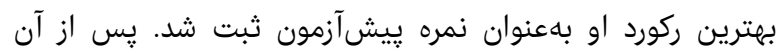

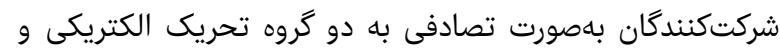

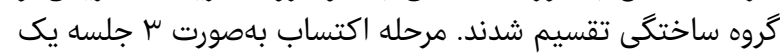

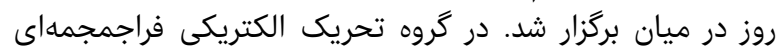

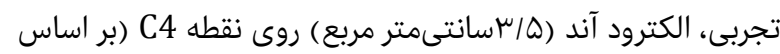

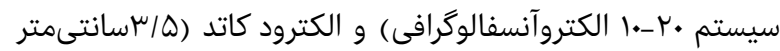

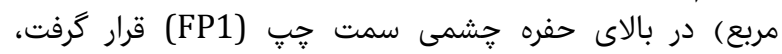

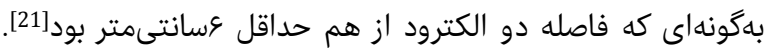

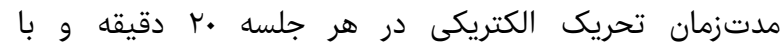

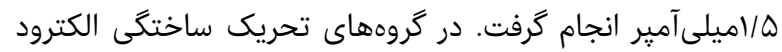

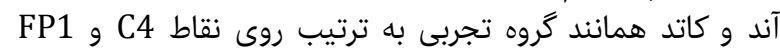

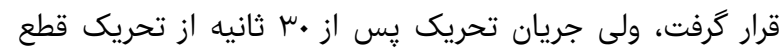

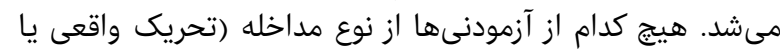

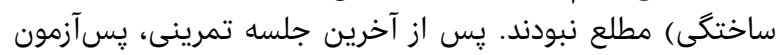

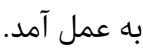

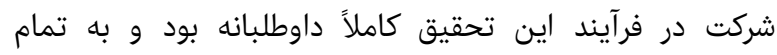

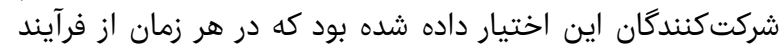

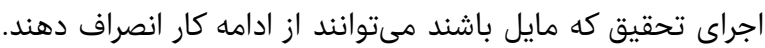

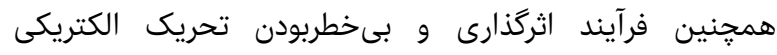
فراجمجمهاى به تمامى شركت آيند أركارى

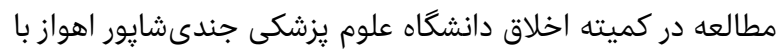

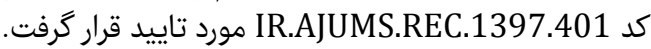

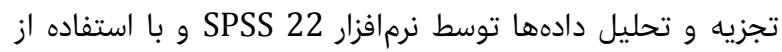

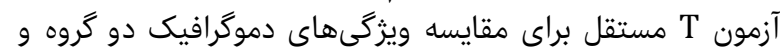

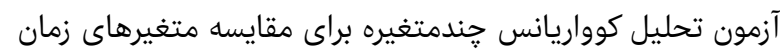

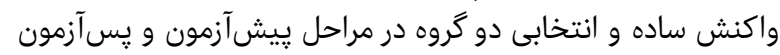
دو گروه انجام شد ساده

\section{يافتهها}

بين دو گروه از نظر ويزگىهاى دموگرافيك، تفاوت معنىدارى

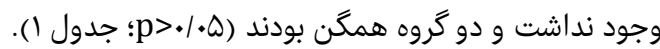

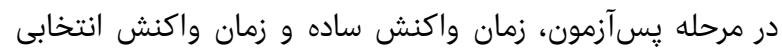

تغيير قابل مشاهده در تابع رفتار يا مولفههاى شناختى از قبيل

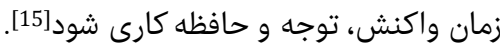

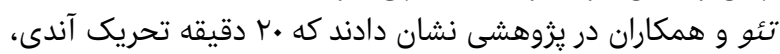

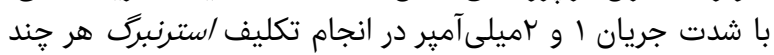

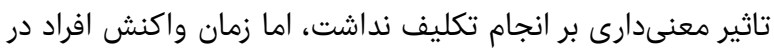

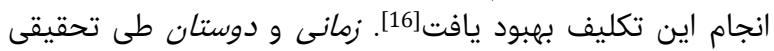

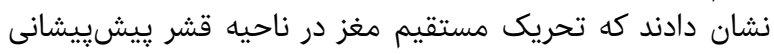

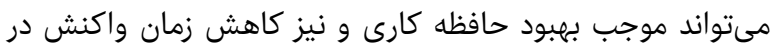

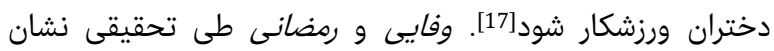

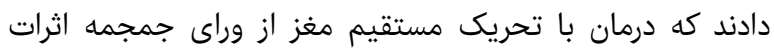

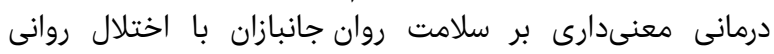

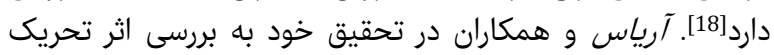

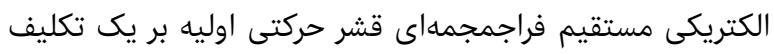

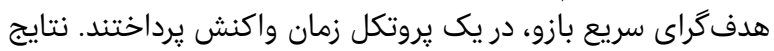

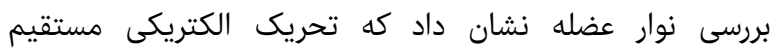

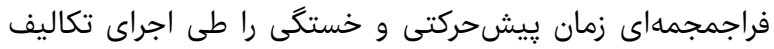

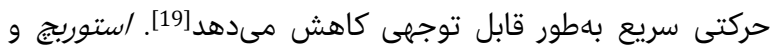

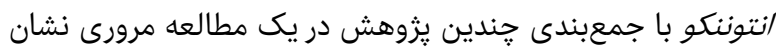

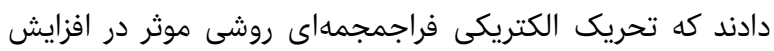

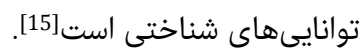

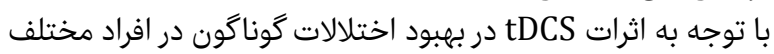

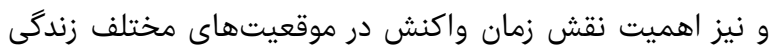

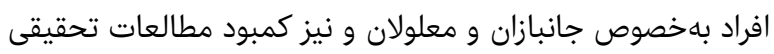

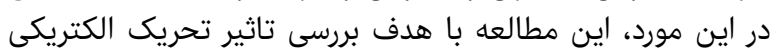

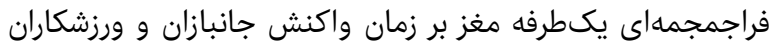

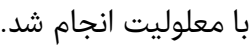

\section{مواد و روشها}

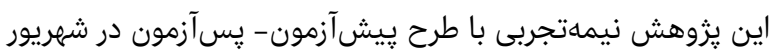

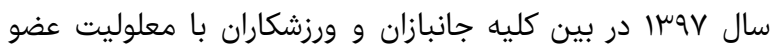

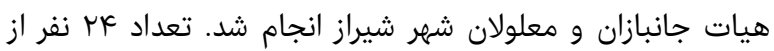

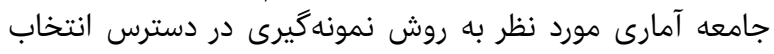

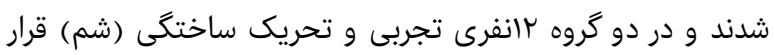

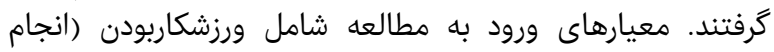

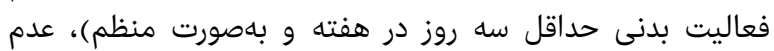

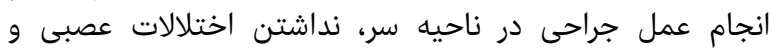

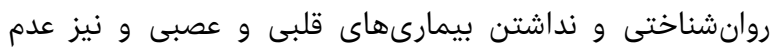

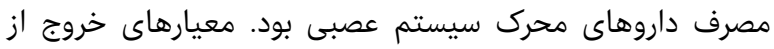

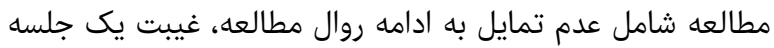

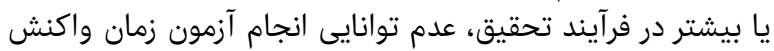

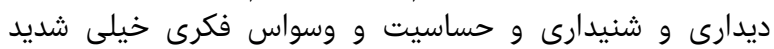

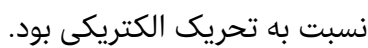

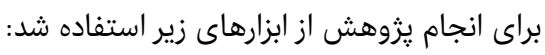

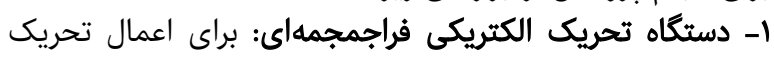

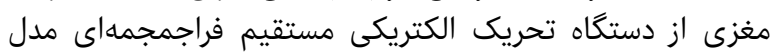

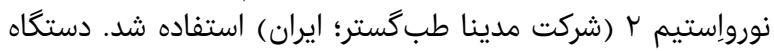

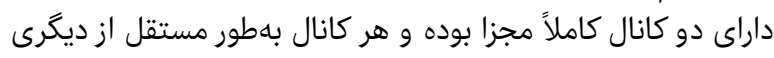

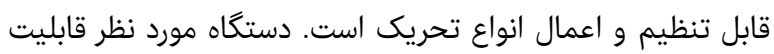

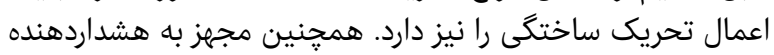

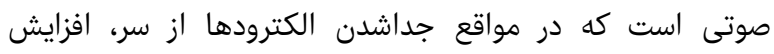

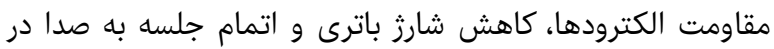

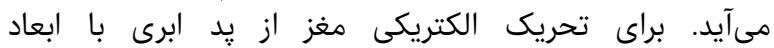




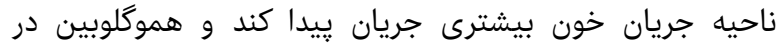

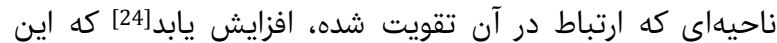

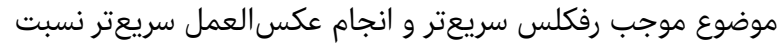

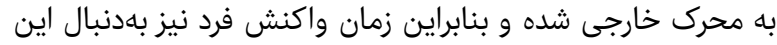

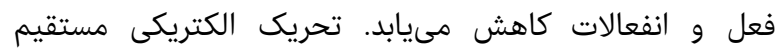

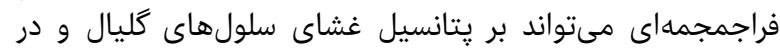

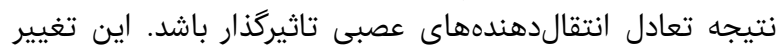

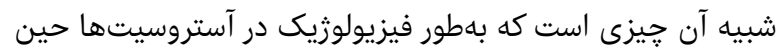

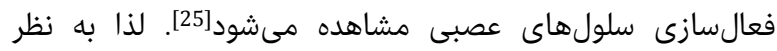

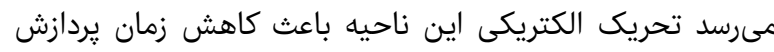

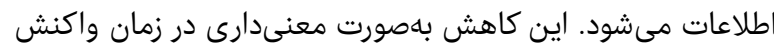

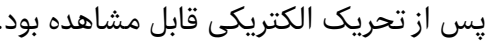

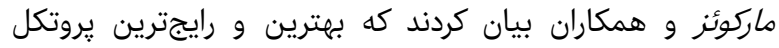

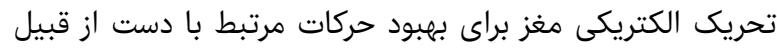

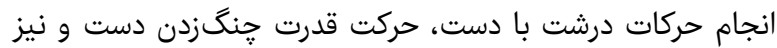

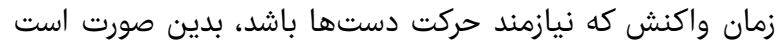

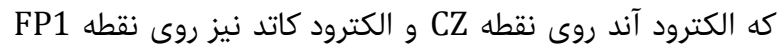

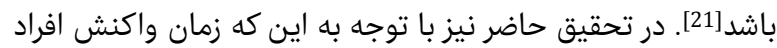

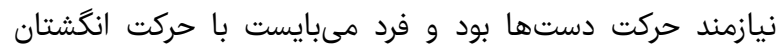

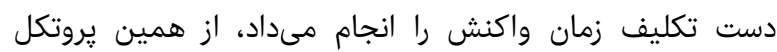

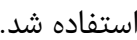

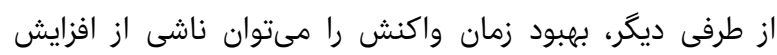

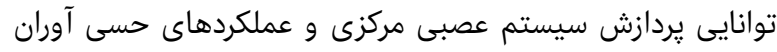

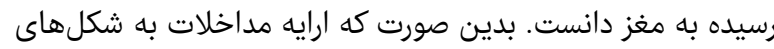

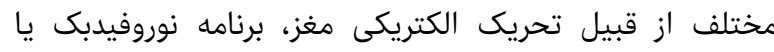

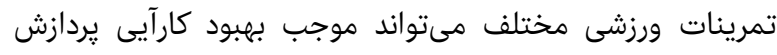

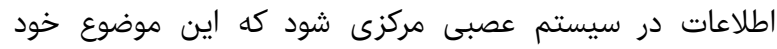

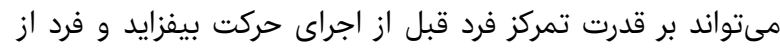

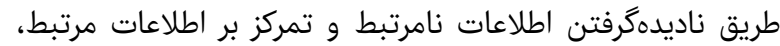

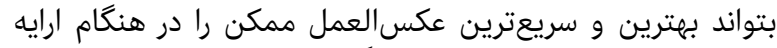

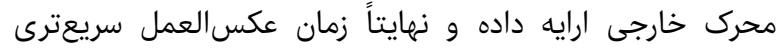

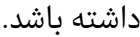

از يافتههاى متناقض بار اين مطالعه مىتوان به مطالعه سوتشك و

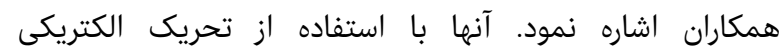

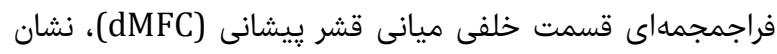

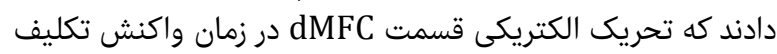

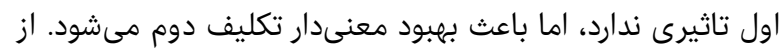

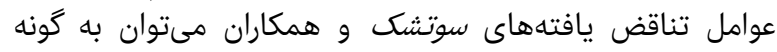

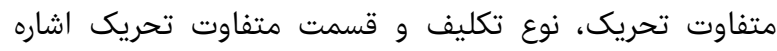

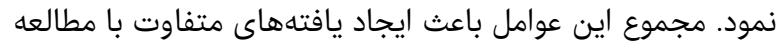

حاضر شده است [26].

وفايى و رضايى طى تحقيقى كه روى جانبازان با بشكلان إنات روانى در

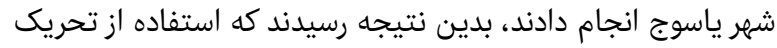

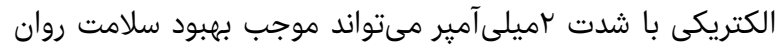

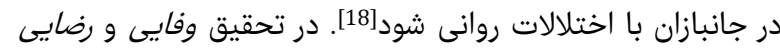

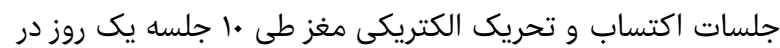

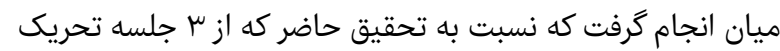

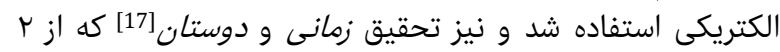

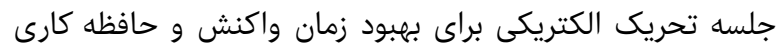

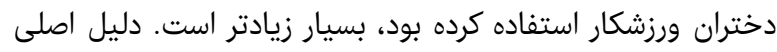

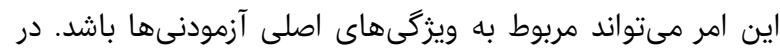
تحقيق حاضر از آزمودنىهاى ورزشكار استفاده شد، در آدردالى حالى كه

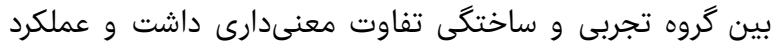

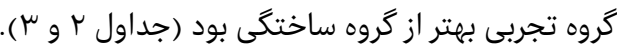

جدول () ميانكَين آمارى ويزَّىهاى دموكرافيك در دو كَروه تجربى (rا نفر) و

\begin{tabular}{|c|c|c|}
\hline كروه ساختى & كروه تجربى & ساحئى (الماى دموكرافيك \\
\hline$\mu q / \cdot \wedge \pm 7 / / \Lambda \Lambda$ & $\psi Y / 77 \pm Y / \varepsilon V$ & سن (سال) \\
\hline $17 Y / 91 \pm 0 / V 0$ & $170 / 0 . \pm 7 / \mu \varepsilon$ & قد (سانتىمتر) \\
\hline $0 \Lambda / \mu \mu_{ \pm N / \gamma}$. & $09 / \cdot \wedge \pm 7 / / \Lambda$ & وزن (كيلوكَرم) \\
\hline
\end{tabular}

\begin{tabular}{|c|c|c|}
\hline يسآزمون & بيشآزمون & متغيرها \\
\hline & \multicolumn{2}{|c|}{ زمان واكنش ساده (هزارم ثانيه) } \\
\hline$r Q \varepsilon / 0 . \pm \mu^{\gamma} \gamma / 97$ & $\mu \mu \tau / \Lambda \mu_{ \pm} \pm \Lambda / \mu{ }^{\mu}$. & كروه تجربى \\
\hline \multirow[t]{2}{*}{$\mu \varepsilon Y / V O \pm{ }^{\mu} T / 71$} & $\mu \varepsilon \vee / Q 1 \pm \mu / / 0$. & 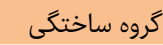 \\
\hline & \multicolumn{2}{|c|}{ زمان واكنش انتخابى (هزارم ثانيه) } \\
\hline $0.1 / \mathrm{rON} \mathrm{VT} / \mu \mu$ & 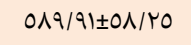 & كروه تجربى \\
\hline OVV/VODTW/MA & $0 \Lambda \cdot 10 . \pm 7 \varepsilon / 11$ & 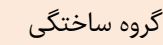 \\
\hline
\end{tabular}

جدول سر) نتايج آزمون تحليل كوواريانس جندمتغيره در بين كروههاى تجربى و ساختخى در زمان واكنش ساده و انتخابى ناري

\begin{tabular}{|c|c|c|c|c|c|c|}
\hline ضريب & معنى سطحى & Fقدار F F & مجذور سوم & & مجذور سوم & متغيرها \\
\hline & & & & & \multirow{2}{*}{\multicolumn{2}{|c|}{ 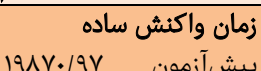 }} \\
\hline ./70 & ||$\ldots \mid$ & r & $191 \mathrm{~V} \cdot / 9 \mathrm{~V}$ & 1 & & \\
\hline . /\& &.$|\ldots|$ & $19 /$ & $q \vee \mu \mid / Y\urcorner$ & 1 & $9 \vee \mu / / R T$ & \\
\hline •/V. & . & $\varepsilon \wedge / 99$ & . & 1 & \multicolumn{2}{|c|}{ زمان واكنش انتخابى. } \\
\hline . $10 \mathrm{r}$ & $\mid . \ldots 1$ & $\mu 7 / 99$ & 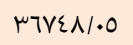 & 1 & 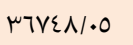 & گروه \\
\hline
\end{tabular}

\section{بحث}

هدف از انجام اين تحقيق بررسى تاثير تحريك الكتريكى مغز بر بران

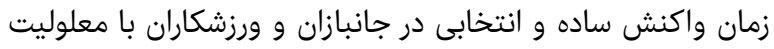

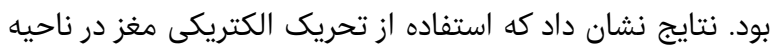

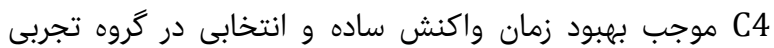

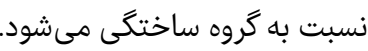

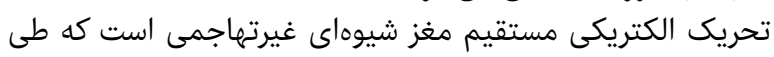

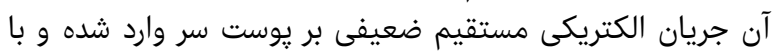

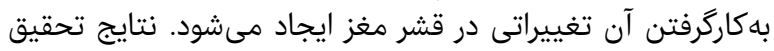

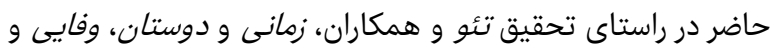

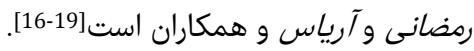

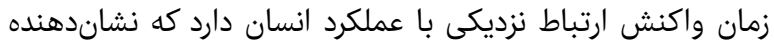

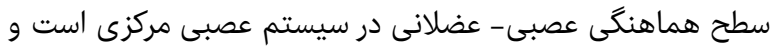

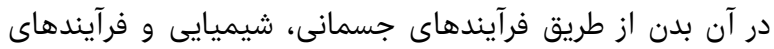

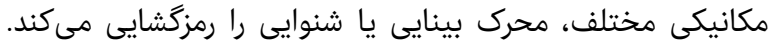

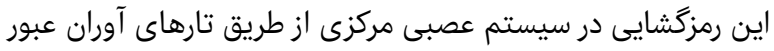

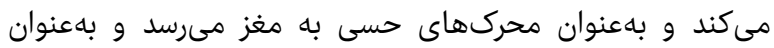

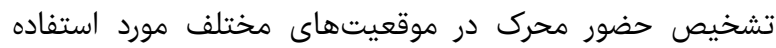

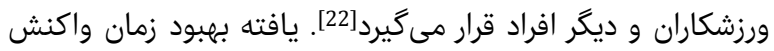

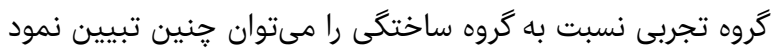

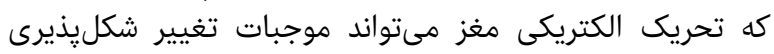

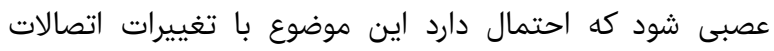

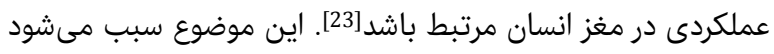

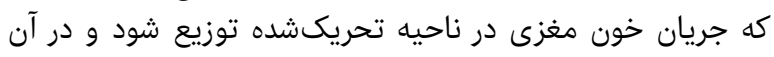


IIr تاثير تحريك الكتريكى فراجمجمهاى يكىطرفه مغز بر زمان واكنش جانبازان و ورزشكاران با معلوليت Bull WHO. 2010;88:815-23.

5- Drescher KD, Foy DW, Kelly C, Leshner A, Schutz K, Litz B. An exploration of the viability and usefulness of the construct of moral injury in war veterans. Traumatology. 2011;17(1):8-13.

6- Shokri B, Zarei M, Sahraei OR. Comparing the mental health of the athletic and non-athletic sensory-disabled people. Q J Soc Work. 2015;4(3):37-43. [Persian]

7- Mohamadtaghi B, Shamsipour Dehkordi P, Hejazi Dinan P. Effectiveness of physical activity on quality of life and pain self-efficacy in veterans and non-veterans with amputations of lower limbs. Iran J War Public Health. 2016;8(2):95-103. [Persian]

8- Robertson EM. The serial reaction time task: Implicit motor skill learning?. J Neurosci. 2007;27(38):10073-5.

9- Alikhani H, Vaez Mousavi M, Mokhtari P. The effect of cognitive and motivational imagery on choice reaction time. World Appl Sci J. 2011;12(6):792-6.

10- Schmidt RA, Lee TD. Motor learning and performance: From principles to application. Champaign: Human Kinetics; 2018.

11- Grouios G. On the reduction of reaction time with mental practice. J Sport Behav. 1992;15(2):141.

12- Clark VP, Coffman BA, Trumbo MC, Gasparovic C. Transcranial Direct Current Stimulation (tDCS) produces localized and specific alterations in neurochemistry: $\mathrm{A}^{1} \mathrm{H}$ magnetic resonance spectroscopy study. Neurosci Lett. 2011;500(1):67-71.

13- Brunoni AR, Amadera J, Berbel B, Volz MS, Rizzerio BG, Fregni F. A systematic review on reporting and assessment of adverse effects associated with transcranial direct current stimulation. Int J Neuropsychopharmacol. 2011;14(8):1133-45.

14- Eslamizade MJ, Behbahanian Sh, Mahdavi SM, Oftadehal M. An introduction to neurotechnologies, transcranial magnetic stimulation and transcranial direct current stimulation: Their applications in the cognitive enhancement and rehabilitation. Shefaye Khatam. 2016;4(2):65-86. [Persian]

15- Strobach T, Antonenko D. tDCS-induced effects on executive functioning and their cognitive mechanisms: A review. J Cogn Enhanc. 2017;1(1):49-64.

16- Teo F, Hoy KE, Daskalakis ZJ, Fitzgerald PB. Investigating the role of current strength in tDCS modulation of working memory performance in healthy controls. Front Psychiatry. 2011;2:45.

17- Zamani G, Doostan MR. The effect of transcranial direct current stimulation on working memory and reactiontime in athlete girls. Neuropsychology. 2017;3(3):51-62. [Persian]

18- Vafaye Sisakht Sh, Ramezani Kh. The effects of transcranial direct current stimulation on mental health of veterans with psychiatric disorders. Shefaye Khatam. 2017;5(2):36-42. [Persian]

19- Arias P, Corral-Bergantiños Y, Robles-García V, Madrid A, Oliviero A, Cudeiro J. Bilateral tDCS on primary motor cortex: Effects on fast arm reaching tasks. PLoS One. 2016;11(8):e0160063.

20- Alboghebish S, Shetab Boushehri N, Danshfar A, Abedanzadeh R. Assiament facilitate and significant interference of Stroop effect on psychological refractory period. Neuropsychology. 2017;2(2):93-106. [Persian] 21- Marquez J, Conley A, Karayanidis F, Lagopoulos J, Parsons M. Anodal direct current stimulation in the healthy aged: Effects determined by the hemisphere stimulated. Restor Neurol Neurosci. 2015;33(4):509-19.

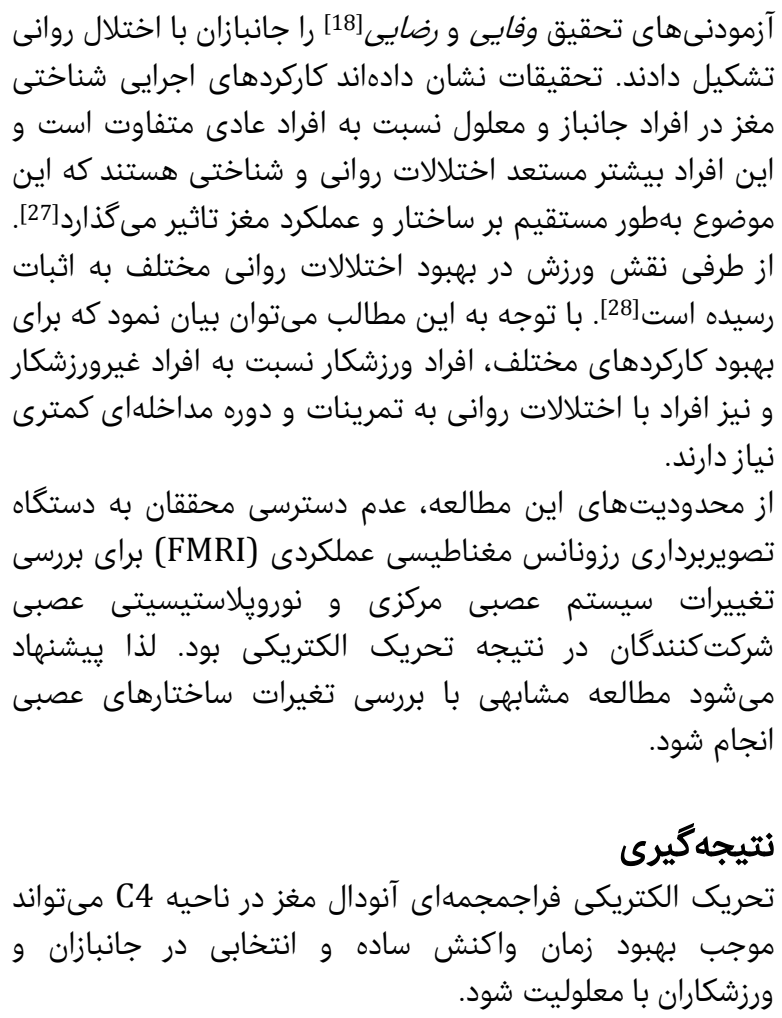

1- Parsaee S, Alboghbish S, Abdolahi H, Alirajabi R, Anbari A. Effect of a period of selected SMR/Theta neurofeedback training on visual and auditory reaction time in veterans and disabled athletes. Iran J War Public Health. 2018;10(1):15-20. [Persian]

2- Kraus WE, Houmard JA, Duscha BD, Knetzger KJ, Wharton MB, Mc Cartney JS, et al. Effects of the amount and intensity of exercise on plasma lipoproteins. N Engl J Med. 2002;347(19):1483-92.

3- Akbarzadeh B, Esmailie A, Rafat MS, Dadashzadeh M. Comparison of self-regulation components between the disabled and veteran athletes and non-athletes. Iran J War Public Health. 2018;10(3):121-25. [Persian]

4- Bedirhan Üstün T, Chatterji S, Kostanjsek N, Rehm J, Kennedy C, Epping-Jordan J, et al. Developing the World Health Organization disability assessment schedule 2.0. 
2011;54(3):2287-96.

25- Ruohonen J, Karhu J. tDCS possibly stimulates glial cells. Clin Neurophysiol. 2012;123(10):2006-9.

26- Soutschek A, Taylor PC, Schubert T. The role of the dorsal medial frontal cortex in central processing limitation: A transcranial magnetic stimulation study. Exp Brain Res. 2016;234(9):2447-55.

27- Tavalaee SA, Habibi M, Asari Sh, Ghanei M, Naderi Z, Khateri Sh, et al. Quality of life in chemical weapon victims 15 years after exposure to mustard gas. J Behav Sci. 2007;1(1):17-25. [Persian]

28- Cohen-Cline H, Turkheimer E, Duncan GE. Access to green space, physical activity and mental health: A twin study. J Epidemiol Community Health. 2015;69(6):523-9.
22- Shelton J, Kumar GP. Comparison Comparison between auditory and visual simple reaction times. Neurosci Med. 2010;1:30-2.

23- Takai H, Tsubaki A, Sugawara K, Miyaguchi Sh, Oyanagi K, Matsumoto $\mathrm{T}$, et al. Effect of transcranial direct current stimulation over the primary motor cortex on cerebral blood flow: A time course study using nearinfrared spectroscopy. In: Elwell CE, Leung TS, Harrison DK, editors. Oxygen transport to tissue XXXVII. New York: Springer; 2016. pp. 335-41.

24- Polanía R, Paulus W, Antal A, Nitsche MA. Introducing graph theory to track for neuroplastic alterations in the resting human brain: A transcranial direct current stimulation study. Neuroimage. 